Review Article

\title{
Opportunities and Challenges in Exploring Indian Non-mulberry Silk for Biomedical Applications
}

\author{
ROCKTOTPAL KONWARH, BIBHAS K BHUNIA and BIMAN B MANDAL* \\ Biomaterial and Tissue Engineering Laboratory, Department of Biosciences \& Bioengineering, Indian \\ Institute of Technology Guwahati, Guwahati 781 039, Assam, India
}

(Received on 07 June 2016; Revised on 08 August 2016; Accepted on 10 November 2016)

\begin{abstract}
Owing to innate desirable features like biocompatibility, mechanical robustness, tunable biodegradability and amenability to multiple formatting, silk (christened as the 'queen of textile') has carved a unique niche in the realm of regenerative medicine. Silkworms, being the major source of silk are generally classified as mulberry and non-mulberry types depending on their feeding habit. Over the years, numerous patents and manuscripts on mulberry based silk for various biomedical applications have been published. In sharp contrast to this, the (immense) potential of the non-mulberry silk for biotechnological applications has been realised quite late. In this article, we have presented the prospects and the recent endeavors to exploit non-mulberry silk (fibroin and sericin) extracted from Antheraea mylitta (tasar), Antheraea assamensis (muga), Philosamia ricini (eri) etc. for fabrication of various formats of biomaterials in applications such as tissue engineering, drug delivery, in vitro tumour modelling, antimicrobial sutures etc. The focus of this article is to highlight the prospective avenues of exploring non-mulberry silk in biomedical domain, as reflected through some of the recent select research works.
\end{abstract}

Keywords: Non-mulberry Silk; Tissue Engineering; Biomaterials; Scaffolds; Drug Delivery

\section{Introduction}

Regenerative medicine stands out as the indispensable demand of the hour in the context of constantly esclating cases of degenerative diseases, skyrocketing medical costs, lack of appropriate organdonors and issues of non-acceptance of transplants (Gurtner et al., 2007; Lieben, 2016). Regenerative medicine (Fig. 1) is an umbrella term encompassing endeavors to bring material science, molecular biology and tissue engineering sciences to assist the body's inherent regenerative potential in vivo (inside the body). This is achieved by the application of growth factors and/or appropriate cell types in vitro (in lab conditions) on suitable scaffold matrices. The choice of material for the scaffolding matrix becomes crucial as it aids in growth and maturation of seeded cells and for further implantation in patients without eliciting any adverse immune reaction (Mason and Dunnill, 2008).

*Authorfor Correspondence: E-mail: biman.mandal@iitg.ernet.in
Amongst others, the scientific fraternity has witnessed the unique journey of silk from the textile and fashion industry to various other stimulating realms, including those of microfluidics-research (Konwarh et al., 2016) and biomedical sciences, in particular, tissue engineering (Melke et al., 2016). Development of silk based biomaterials/scaffolds is envisaged to assist in tissue replacement, repair and regeneration as well as understanding human physiology, pathophysiology and toxicology. Such scaffolds provide a three dimensional structure with tunable physicochemical properties including pore size, porosity, swelling capacity, mechanical strength etc. (Abbott and Kaplan, 2016).The scaffolds assist in the cell proliferation, migration and nutrient or waste material exchange. Fabrication of these biomaterials is streamlined to explore the innate attributes of silk including resilience, biocompatibility and tunable biodegradation profile (Li et al., 2015). Siphoning the use of Indian endemic silk into the niche of 


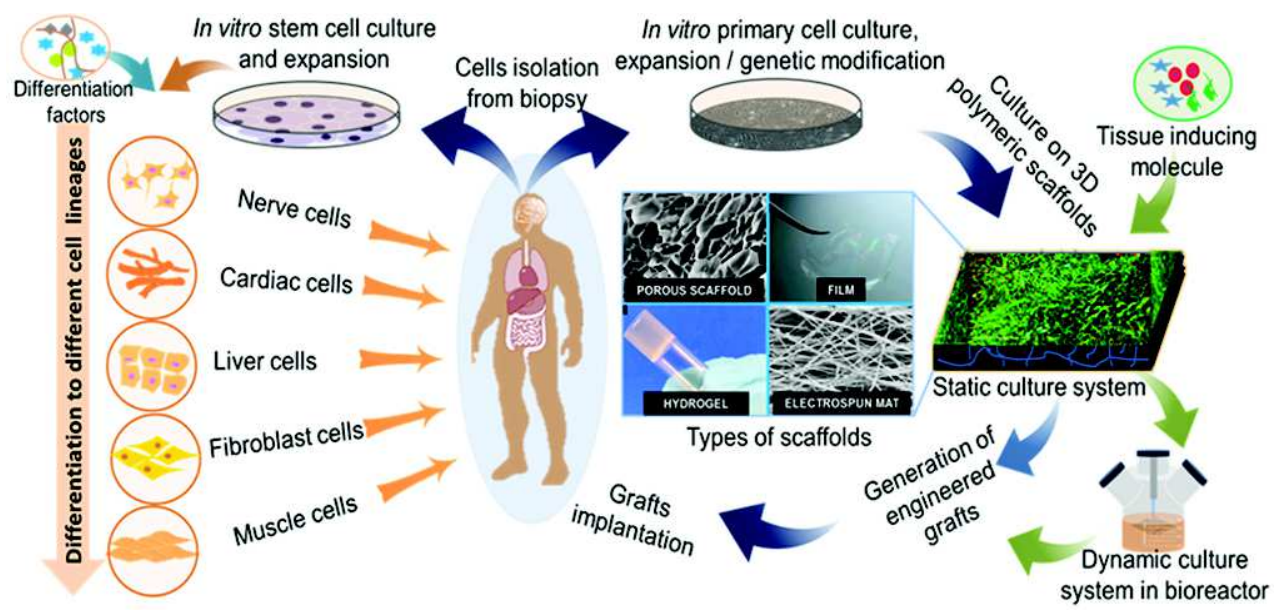

Fig. 1: Schematic representation of the concept of tissue engineering and regenerative medicine

regenerative medicine is expected to open up avenues to address some of the pressing biomedical issues.

In various pockets of our country, silkworms, the lepidopterans belonging to the families of Bombyciidae and Saturniidae have been commercially exploited for production of silk. Silkworms are classified into mulberry (e.g., Bombyx mori) and non-mulberry (e.g., Antheraea mylitta, Antheraea assamensis, Philosamia ricini) types depending on the feeding habit. The mulberry silkworms, B. mori (belonging to Bombyciidae) feed on the leaves of mulberry plants and contribute about $90 \%$ of global silk production. There exists plethora of reports on the protocols and associated challenges of cultivating the domesticated mulberry silkworm as well as studies in the perspective of its protein chemistry, genome and exploitation in various domains including tissue engineering, drug delivery and nanobiotechnology (Sangkert et al., 2016; Wang et al., 2016; Liu et al., 2016; Patil et al., 2016). Some of the representative works involving $B$. mori silk biomaterials are presented in Fig. 2. As for evidence, a simple prototype device was fabricated for directional freezing of $B$. mori, circumventing the issue of heterogeneity in alignment and pores in laminar scaffolds for survival of chondrocytes and differentiation of bone marrow stem cells (Mandal et $a l ., 2013)$. With the goal of engineering bone lamellae, the alignment and osteogenic differentiation of MSCs on patterned B. mori silk films have been documented (Tien et al., 2012). Interestingly, high compressive strength ( $13 \mathrm{MPa}$ in hydrated state) 3D scaffold based on $B$. mori silk protein-protein interfacial bonding has been fabricated for bone tissue engineering (Mandal et al., 2012). Three layered wedge shaped $B$. mori silk scaffold system has been projected for knee meniscus grafts (Mandal et al., 2011). Besides, RGD-coupled, porous, patterned, mechanically robust and transparent $B$. mori silk films were fabricated to mimic corneal stromal tissue architecture and hierarchy (Gil et al., 2010). Prior work also testifies the successful application of $B$. mori fibroin/gelatin multilayer biocompatible films for controlled release of different compounds including trypan blue, FITC-inulin and FITC-BSA (Mandal et al., 2009a). Recently, the prospects of blending silk fibroin and human hair-derived keratin have been documented for wound healing application in skin tissue engineering (Bhardwaj et al., 2015).

On the other hand, Antheraea and Philosamia, the saturniid silkworms, are two major sources of wild/non-mulberry silk in India (Kundu et al., 2012). These silk varieties are symbolic of the dynamic and rich cultural heritage of different serizones of India. To cite for evidence, the resplendence and the picturesque floral motifs of a pair of muga (the golden yellow silk, capped with the Geographical Indication, GI status) (Phukan, 2012) mekhela chadar (a traditional dress worn by Assamese women) reverberate the creative genius of the weavers and the toil of the sericulturists of the state of Assam. At this juncture, it would be relevant to mention that research on various non-mulberry silk biomaterials has geared up in various institutes all over the world. Amongst others, endeavours are also underway to explore north-east Indian silk based biomaterials using 
innovative approaches like electrospinning and others for regeneration of a plethora of tissues. These biomaterials may not only be used for tissue engineering but also as 3D disease tissue models and delivery of cells and drugs.
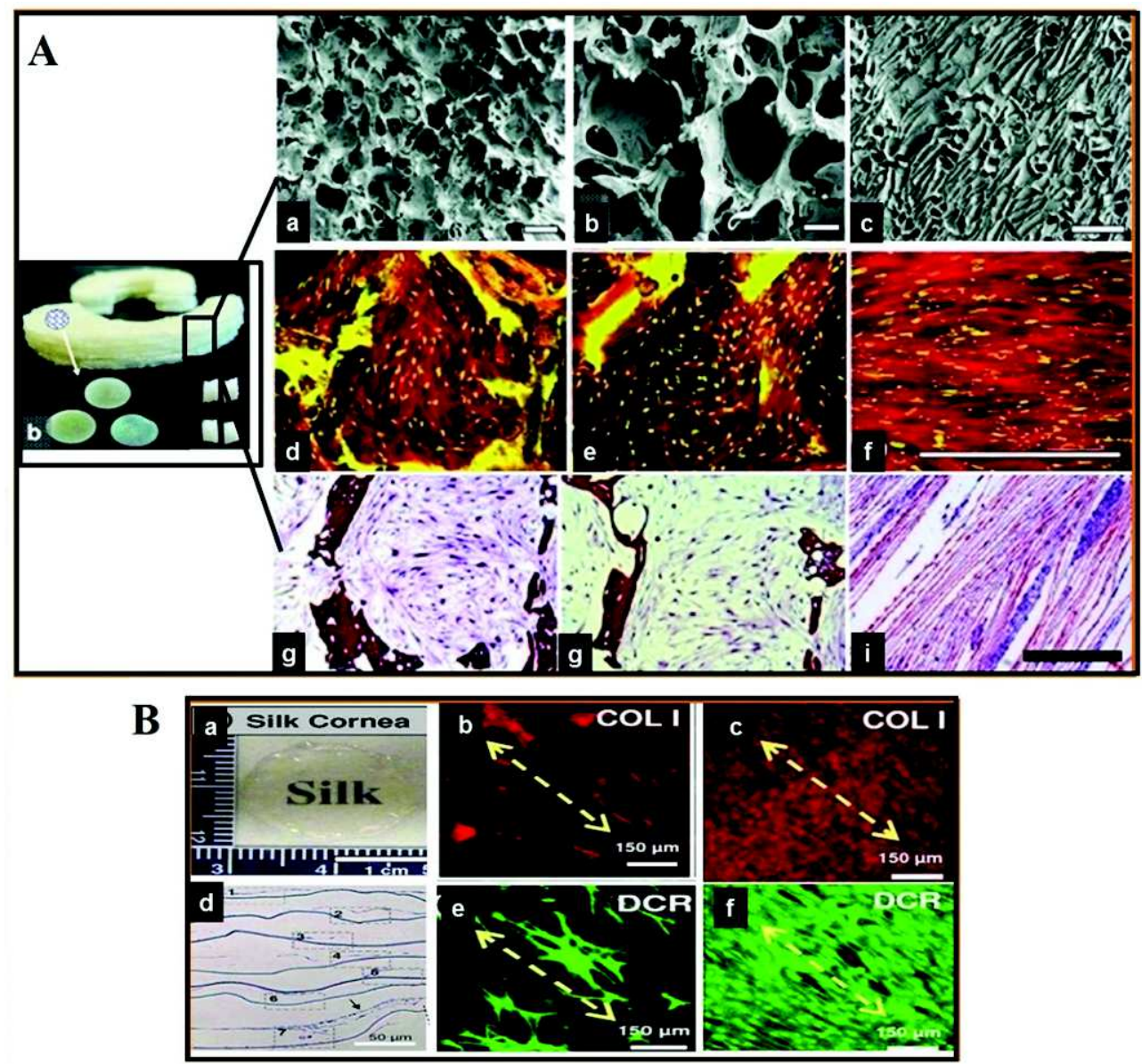

C

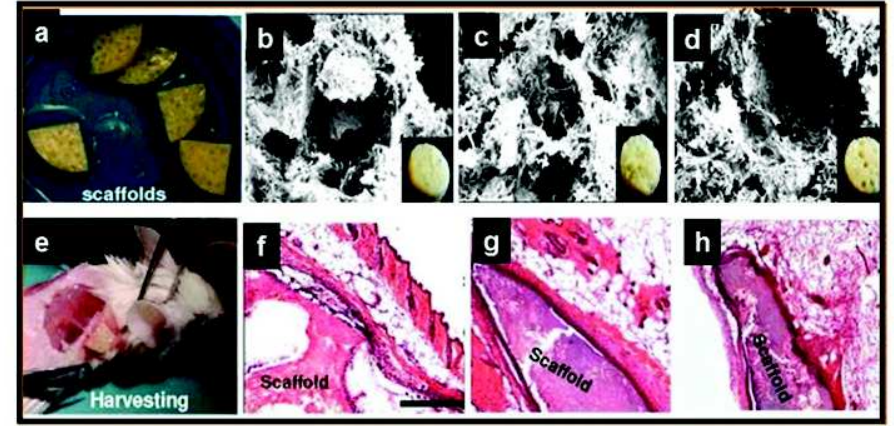

Fig. 2: [A] Silk scaffolds for functional meniscus tissue engineering; (a-c) scanning electron micrograph of fabricated three layers, (a) top layer, (b) middle layer and (c) bottom layer, (d-f) Confocal images of hMSCs on individual scaffold layers in chondrogenic medium after 14 days, (d) top layer, (e) middle layer and (f) bottom layer, (g-i) saffranin $O$ staining for sGAG secreted in scaffolds after 28 days (g) top layer, (h) middle layer and (i) top layer. Reproduced with permission from (Mandal et al., 2011) ${ }^{\circledR} 2011$ Elsevier. [B] Multi-layered silk films for corneal tissue engineering; (a, d) stacked films with cells for corneal construct and $H \& E$ staining after 1 week, (b, c) immunostaining for collagen type I of (b) without RGD pattern film and (c) RGD modified pattern film, (e, f) immunostaining for decorin of (e) pattern film without RGD and (f) RGD modified pattern film. Reproduced with permission from (Gil et al., 2010) ${ }^{\circ} 2010$ Elsevier. [C] High strength silk scaffold for bone tissue engineering; (a) fiber reinforced high strength scaffolds, (b-d) scanning electron microscopy images of different length fiber reinforced scaffolds, (b) small fiber, (c) middle length fiber and (d) large fiber, (e) harvesting of implanted scaffold, (f-h) histological analysis for in vivo responses of transplanted scaffolds after 4 weeks, (f) small fiber (g) middle length fiber and (h) large fiber. Figure adopted from (Mandal et al., 2012) 
Therefore, we have tried to compile the recent scientific endeavors on the exploration of Indian nonmulberry silk in the biomedical domain in the present article. We start off with a short note on the nonmulberry silk worms and their life cycle. Then, a section is dedicated to the discussion of the major proteins and signature-sequences, relevant to biomedical engineering while keeping the prime focus on the plethora of biomedical applications and the associated intricacies along with possible solutions in exploring non-mulberry silk.

\section{A note on non-mulberry silk worms}

\section{Diversity}

A wide variety of non-mulberry silk worms (Kundu et al., 2012) has been identified in various geographical locations and the exploitation of the same has been reported for harvesting silk. These are summarized in Table 1.

Table 1: Key features of major non-mulberry silk worms (adapted from Kundu et al., 2012)

\begin{tabular}{|c|c|}
\hline Non-mulberry silkworm & Key features \\
\hline Tasar (Antheraea mylitta) & $\begin{array}{l}\text { Endemic to Indian subcontinent- Uni-, bi-, or tri-voltine; depending on geo-ecological } \\
\text { conditions } \\
\text { Feeds mainly on the leaves of Shorea robusta and Terminalia trees } \\
\text { Most dominant, largest in size and highest silk producing capacity among all non-mulberry } \\
\text { silk worms }\end{array}$ \\
\hline Chinese (Oak) tasar (Antheraea pernyi) & $\begin{array}{l}\text { Predominant in southern China, also found in north-eastern part of India. Uni, or bi- } \\
\text { voltine } \\
\text { Mostly feeds on the leaves of oak trees } \\
\text { The black hatchlings turn to pale yellowish green post first sheds of skin }\end{array}$ \\
\hline Muga (Antheraea assamensis) & $\begin{array}{l}\text { Endemic to north-eastern zone of India, particularly in the Brahmaputra valley and } \\
\text { adjoining hills of Assam } \\
\text { Multi-voltine and semi-domesticated } \\
\text { Polyphagous and feeds on the leaves of Litsea, Machilus, Cinnamomum, and Actinodaphne } \\
\text { spp. trees } \\
\text { Produces golden yellow color yarn } \\
\text { Susceptible to various diseases, pest and predators } \\
\text { Lesser heterogeneity and sexual dimorphism with regard to colour pattern in contrast to } \\
\text { A. mylitta }\end{array}$ \\
\hline Eri (Philosamia ricini) & $\begin{array}{l}\text { Found in north-eastern part of India, China, Japan and also across the palaearctic and } \\
\text { Indo-Australian biogeographic regions } \\
\text { Multi-voltine and domesticated } \\
\text { Primarily feeds on the leaves of castor tree (Ricinus communis L.) } \\
\text { Produces spun silk }\end{array}$ \\
\hline Fagaria (Attacus atlas) & $\begin{array}{l}\text { Predominant across the Malay archipelago (Indonesia, Singapore and Philippines) } \\
\text { Often consider as the largest silkworm in terms of total wings span } \\
\text { Bi-voltine } \\
\text { Polyphagous; feeds on the mature leaves and rarely attacks the developing leaves } \\
\text { Yarn exhibits higher tensile strength than muga or eri }\end{array}$ \\
\hline Shashe (Gonometa postica) & $\begin{array}{l}\text { Endemic to the Savannas of eastern and southern Africa } \\
\text { Uni-, bi-, or multi-voltine } \\
\text { Feeds on the leaves of Burkea africana, Acacia erioloba, A. melifera, A. tortili and } \\
\text { Brachystegia spp. } \\
\text { The mature larvae is protected by spine, a silken cocoon to immobilize the pupa } \\
\text { The moth and the cocoons are dimorphic in nature; females are twice the size of males }\end{array}$ \\
\hline Cricula (Cricula trifenestrata) & $\begin{array}{l}\text { Distributed across the Indian sub-continent to Philippines, Java and Sulawesi } \\
\text { Bi- or multi-voltine depending on geo-ecological conditions } \\
\text { Smallest silkmoth among all non-mulberry silkworms } \\
\text { Polyphagous; feeds on the leaves of mainly fruit trees e.g., Prunus, Malus, Pyrus, } \\
\text { cashew and so on } \\
\text { Produces golden perforated cocoons }\end{array}$ \\
\hline
\end{tabular}




\section{Life Cycle of Non-mulberry Silk Worm}

Duration of life cycles of different non-mulberry silkworms varies with the species-type and climate. The various species show different physiological, morphological and growth characters depending on feeding behavior as highlighted in the above table. Beside this, the number of cycles per year (termed as voltinism) depends on the strain as well as environmental conditions like temperature. The life cycle of the non-mulberry silk worms can be classified into the following stages (Kundu et al., 2012) (Fig. $3)$.

- $\quad$ Egg: On advent of favorable conditions, post copulation, adult female moth lays fertilized eggs on the surface of leaves of the host plant or in its vicinity.

- Larva: Post hatching, the larvae, the elongated caterpillars, begin to consume the host leaves and grow. It is the only feeding stage in the life cycle. During larval stage, they undergo different molting phases. The interval between the phases is termed as 'instar'. Wild non-mulberry silkworms are tetra-molters with five instars. The duration of instars and molting periods vary from 4 to 15 days and 24 to $36 \mathrm{~h}$ respectively. In the final larval stage, the fifth instars stop feeding, shorten their body size and start to spin cocoon.

- Pupae: The encapsulating cocoon, majorly made of silk proteins confers protection from adverse environmental conditions. Pupation takes place within the cocoon. The larva remains inside it until favorable conditions return.

- Adult: Under favorable circumstances, the pupa releases the enzyme cocoonase that digests and softens the cocoon layers, and emerges out of the cocoon as an adult moth. The adults undergo copulations and lay eggs, thereby initiating the next generation of the cycle

\section{Major Proteins and 'Signature-Sequence' Germane to Tissue Engineering}

Silk worm proteins- fibroin and the gummy sericin, exploited for biomedical applications (as highlighted later in this article) may be extracted from either cocoons or silk glands. Silk exists as highly concentrated solution with $\alpha$-helix and random coil conformation in glands. During biospinning, a highly specialized process, silkworms extrude protein through a spinneret with rapid elongation flow that converts the random coils to $\beta$-sheets. This process (dictated by microfluidics principle) is associated with various factors like exposure to air, shear stress and $\mathrm{K}+$ ions concentration (Chae et al., 2013). As literature abounds on exploitation of mulberry silk (B. mori) for tissue engineering applications, it would be relevant to place a note on the same prior to specifically discussing about non-mulberry silk.

B. mori fibroin consists of heavy $(\mathrm{H}, 391.367$ $\mathrm{kDa})$ and light $(\mathrm{L}, 25 \mathrm{kDa})$ polypeptide chains, linked by a disulfide bond between the Cys-c $20\left(20^{\text {th }}\right.$ residue from the $\mathrm{COO}^{-}$terminus) of the H-chain and Cys172 of L-chain (Inoue et al., 2000). A glycoprotein, P25 $(25 \mathrm{kDa})$ is attached non-covalently to both the chains. The primary structure of B. mori fibroin consists of multiple repeats of AGSGAG which make up 55\% of the total fibroin, conferring crystallinity to the structure. More specifically, H-chain consists of twelve domains, each domain comprising of hexapeptide sub-domains including GAGAGY, GAGAGS, GAGYGA or GAGAGA (Zhou et al., 2001). L-chain consists of relatively high amount of leucine, isoleucine, valine and acidic amino acids. The structural changes from Silk I (prior to crystallization) to Silk II (crystallized) occurs exclusively in crystalline region post spinning or alcohol treatment that induce $\beta$-sheet formation in between the repeating units. It has been reported that $B$. mori fibroin consists of mainly $30 \%$ alanine, $46 \%$ glycine, $12 \%$ serine, $5.5 \%$ tyrosine, $1 \%$ aspartic and glutamic acid, and 2.2\% isoleucine, leucine and valine (Gupta et al., 2015). Five polypeptides ranging from 80 to $310 \mathrm{kDa}$ constitute the gland sericin while polypeptides of 400 $\mathrm{kDa}, 250 \mathrm{kDa}, 150 \mathrm{kDa}$ and $24 \mathrm{kDa}$ make up cocoon sericin. The amino acid compositions of mulberry and different non-mulberry silk fibroin are compared in Table 2.

We now specifically present the discussion on the non-mulberry silk proteins in the following section.

\section{A. mylitta}

Homodimeric gland fibroin of $\sim 395 \mathrm{kDa}$ (each monomer being $\sim 197 \mathrm{kDa}$ ) has been determined in the tasar silk variety. 5 different fractions $(30 \mathrm{kDa}-$ $>200 \mathrm{kDa}$ ) make up gland sericin while 3 fractions 


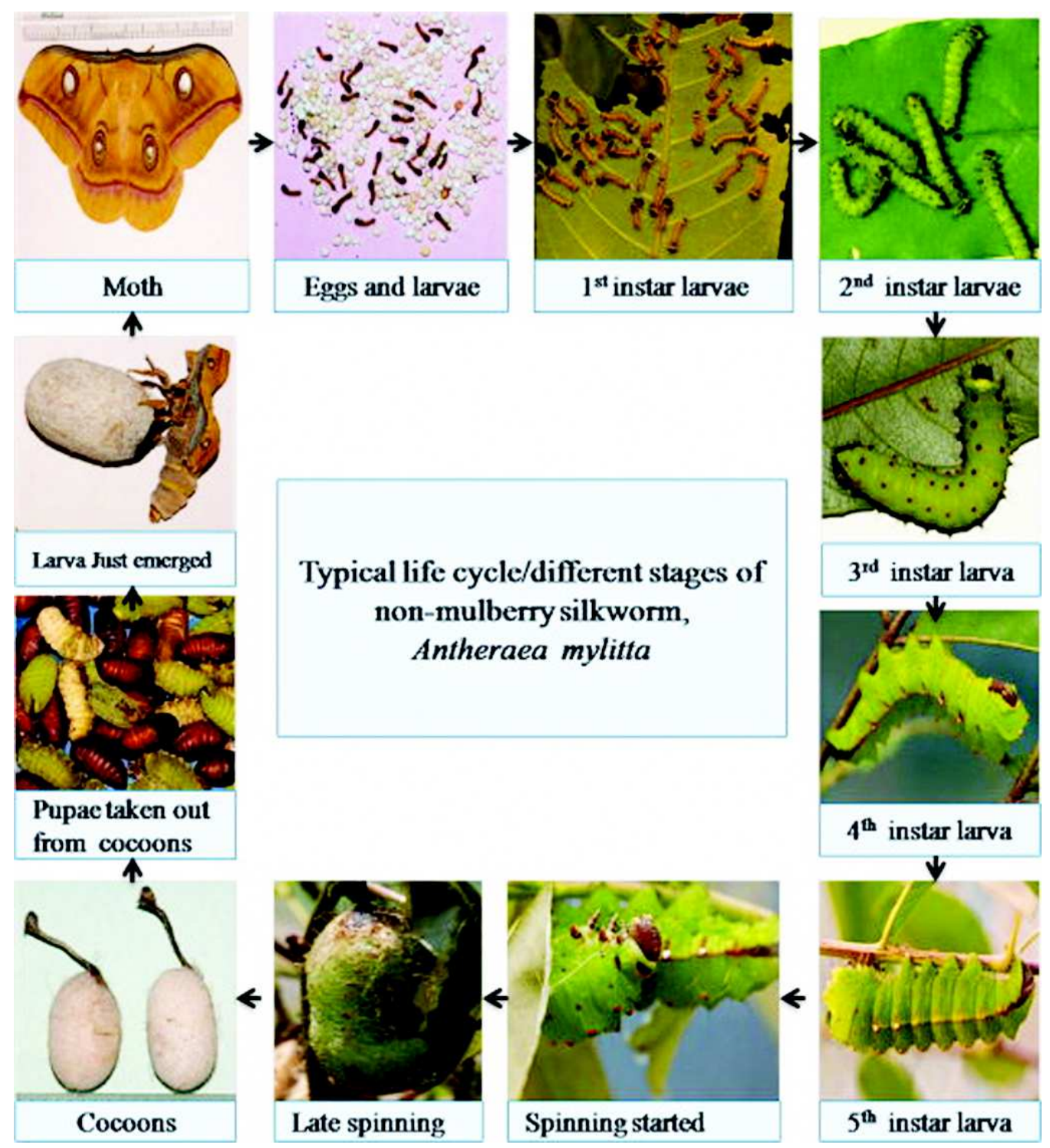

Fig. 3: Typical life cycle/different stages of non-mulberry Indian tropical tasar silkworm, Antheraea mylitta as an example. Figure adapted from (Kundu et al., 2012) ${ }^{\circledR} 2012$ Wiley Periodicals, Inc.

Table 2: Compositional abundance of different amino acids of mulberry and non-mulberry silk fibroin (Gupta $e t$ al., 2015; Sen and Babu, 2004; Reddy and Yang, 2010)

\begin{tabular}{lcccc}
\hline Amino acids & \multicolumn{4}{c}{ Amino acid compositional abundance (mol \%) } \\
\cline { 2 - 5 } & B. mori & A. assama & P. ricini & A. mylitta \\
\hline Ala & 30.2 & 42.5 & 47.9 & 27.7 \\
Gly & 45.9 & 28.9 & 31.4 & 30.89 \\
Ser & 12.1 & 10.2 & 5.1 & 13.72 \\
Arg, Lys, His & 0.6 & 3.9 & 3.61 & 5.02 \\
Tyr & 5.3 & 4.9 & 5.56 & 9.37 \\
Glu, Asp & 1.0 & & 4.32 & 1.26 \\
Ile, Leu, Val & 2.2 & 1.2 & 0.8 & 2.94 \\
\hline
\end{tabular}

(70 kDa, $200 \mathrm{kDa}$ and $>200 \mathrm{kDa}$ ) were found to constitute the cocoon sericin (Kundu et al., 2012). $O$-linked glycosylation is observed in A. mylitta silk fibroin (Datta et al., 2001). The $O$-linkage conforms to a core 1 mucin-type oligosaccharide structure which consists of at least eight monosaccharide residues linked to the protein backbone by $N$-acetyl galactosamine moiety.

\section{P. ricini}

P. ricini fibroin protein is constituted of $97 \mathrm{kDa}$ (with compositional abundance of glutamic acid) and $45 \mathrm{kDa}$ polypeptides connected through a disulfide bond while 
the cocoon- sericin comprises of a $66 \mathrm{kDa}$ molecule (Ahmed et al., 2004). The basic repeating unit of $P$. ricini silk fibroin (SF) is (alanine) ${ }_{12-13}$ which is similar to spider (major ampullate) silk that possesses (alanine) $)_{5-6}$ as repeating unit.

\section{A. assamensis}

Two fractions of $220 \mathrm{kDa}$ and $20 \mathrm{kDa}$ have been reported for A. assamensis fibroin while a single fraction of $66 \mathrm{kDa}$ constitute the sericin (Kundu et al., 2012). The primary structure of $A$. assamensis SF comprises of $42.5 \%$ alanine, $28.9 \%$ glycine, $10.2 \%$ serine and $5.5 \%$ tyrosine (Gupta et al., 2015). The secondary structure of the muga silk fibroin possesses three main motifs: A-motif, consisting of alanine residues; G-motif, primarily composed of glycine residues; and R-motif that contains arginine residues. The A-motif contains poly-alanine stretches of (alanine) $)_{5-15}$ and is responsible for the crystallinity of the protein. One of the unique features of polyalanine stretches of A. assamensis SF is the absence of any interspersing amino acid unlike their counterparts with $3-4 \%$ serine residues in other members of the saturniid family.

Various amino acid ratios of different nonmulberry SF have been presented in Table 3. In a recent endeavor to correlate the physical properties and sequence in silkworm fibroin (Malay et al., 2016), strictly-defined structural transitions of the nonmulberry silk fibroin in the yielding and strain hardening events (tensile deformation and thermal assessments) were worthwhile to note in comparison to that of $B$. mori. It was predicted that modular repeats of the saturniid sequences tend to produce structures that respond in a concerted fashion in contrast to heterogeneous structures of the mulberry counterpart.

The presence of intrinsic cell binding RGD sequences within non-mulberry silk fibroin (NMSF)

Table 3: Various amino acid ratios of different nonmulberry silk fibroin (Reddy and Yang, 2010)

\begin{tabular}{lcccc}
\hline Ratios & A. mylitta & P. ricini & A. assamensis & B. mori \\
\hline Basic/acidic & 0.97 & 1.30 & 1.24 & 0.65 \\
$\begin{array}{l}\text { Hydrophilic/ } \\
\text { hydrophobic }\end{array}$ & 0.44 & 0.35 & 0.38 & 0.28 \\
$\begin{array}{l}\text { Bulky/non-bulky } \\
\text { side group }\end{array}$ & 0.18 & 0.24 & 0.28 & 0.17 \\
\hline
\end{tabular}

confers special niche to the resilient, biocompatible and biodegradable biomaterials based on the latter in the domain of tissue engineering (Bellis, 2011). We have previously shown desirable cell response in terms of good cytoskeleton organization, actin development, cell spreading, and strong binding to substratum using A. mylitta fibroin protein films having RGD sequences in comparison to $B$. mori counterpart (Mandal et al., 2010). Furthermore, mechanical robustness of the NMSF reported in terms of its higher tensile strength than mulberry SF has been bracketed together with the lesser heterogeneity and orderly arrangement of non-polyalanine repeats in higher number, tighter poly-alanine $\beta$-crystals that are devoid of non-alanine residues and lesser amount of polar residues in the protein main chain (Datta et al., 2001).

\section{Dissolution and Extraction of Non-mulberry Silk Fibroin}

During SF extraction from mulberry silk worm cocoons (Mandal et al., 2011); there occurs a disintegration of $\beta$-sheet of the biopolymer, resulting in random coils and alpha helical structures. The $\beta$ sheet structures are re-inducted post biomaterial fabrication for stabilization through techniques like water vapour annealing or ethanol treatment. However, solubilization of non-mulberry silk cocoon is a formidable task due to its hydrogen bonds and hydrophobic nature. Use of chaotropic agents like lithium bromide ( $\mathrm{LiBr}$ ) (conventionally used for extracting $B$. mori silk fibroin) to solubilize nonmulberry cocoons is less effective and results in less yields. Posterior silk gland is an alternative source of silk protein. The basic difficulty for isolation of gland proteins is the rapid aggregation and precipitation on exposure to salt. It can dissolve in $8 \mathrm{M}$ urea, but has the tendency to aggregate when stored at $4^{\circ} \mathrm{C}$. Use of lithium dodecyl sulfate (LDS) is one successful way to dissolve glands protein (Datta et al., 2001). However, lithium salts known to affect metabolism, neural communication and cell proliferation (Phiel and Klein, 2001) should be removed completely from proteins prior to their application in biomedical and tissue engineering fields. In this context, the use of an anionic detergent, sodium dodecyl sulfate (SDS) (Mandal and Kundu, 2008) was documented for the solubilization and regeneration of NMSF from glands (Fig. 4). 


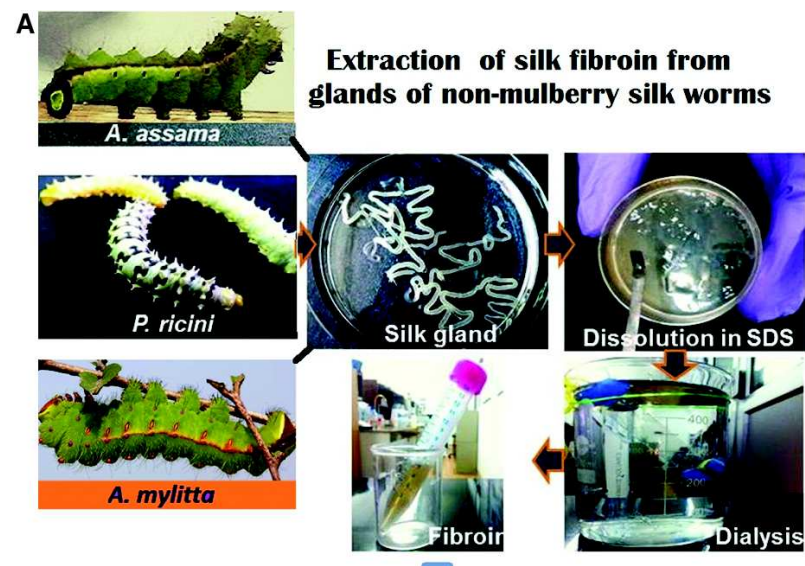

Different formats of silk fibroin based biomaterials

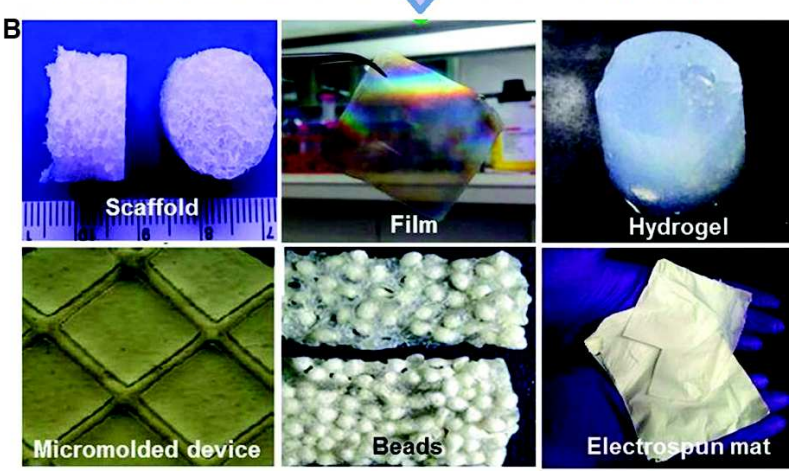

Fig. 4: Procedure followed for silk gland fibroin protein isolation from matured $5^{\text {th }}$ instar larvae of nonmulberry silkworms and different fabricated matrices. The image of the micromolded device is reproduced with permission from (Mandal et al., 2009b) ${ }^{\odot 2008}$ Springer. The image of the beads is reproduced with permission from (Mandal and Kundu, 2009a) ${ }^{\odot 2009}$ Elsevier

A critical micelle concentration $(6 \mathrm{mM})$ of SDS is needed to maintain micellar orientation and structure entrapping fibroin in the hydrophobic core. SDS solubilizes the protein and hinders $\beta$-sheet formation by increasing interactions between hydrophilic blocks of protein. This procedure also overcomes the innate limitations related to $B$. mori silk fibroin isolation. In the same token, the process facilitates high yield of the protein $(\sim 1 \mathrm{~g} /$ matured fifth instar larva). Post hydrolysis, the regenerated, non-bioengineered gland silk fibroin primarily consists of random coils and alpha helix (as in the native state). $\beta$-sheets in non-mulberry silk fibroin biomaterials are induced post exposure to water vapor or ethanol, amongst others.

\section{Non-mulberry Silk for Biomedical Engineering}

Non-mulberry silk may be fabricated into different formats including 2D films, hydrogels, 3D scaffolds, nanofibrous mats and particles that could be used as biocompatible and affordable biomaterials (Kundu et $a l ., 2012)$. The compilation presented here sums up some of the recent endeavours to explore NMSF in tissue engineering and other biomedical applications including drug delivery, sutures and antimicrobial coatings along with in vitro tumour models. One or the various other fabricated formats have been adroitly used for these applications.

\section{Tissue Engineering Applications}

Films/membranes: The escalating number of cardiovascular diseases has become the cardinal cause of death worldwide. As a plausible approach to address this, a topical report (Gupta et al., 2016) testifies the endeavours to exploit mulberry (Bombyx mori) and Indian endemic non-mulberry silk protein (Philosamia ricini and Antheraea assamensis) through sequential rolling of cell-laden patterned films for the facile fabrication of an affordable and biodegradable vascular graft. The protocol circumvents the conventional maturation step of grafts in pulsatile bioreactor. Support to vascular cell growth, induction of cellular alignment and functional cellular phenotype, with minimal in vivo immunogenic response were documented for the fabricated graft, exhibiting desirable mechanical features. It withstood the physiological blood pressure as evinced by the burst strength ranging 915-1260 mm Hg. Further in vivo assessments would pave the route for clinical translation of the fabricated biomimetic small diameter vascular graft, envisaged to be available as 'off the shelf' product for replacement of occluded/diseased coronary artery and arteriovenous fistula for hemodialysis access.

Another promising endeavor of recent times is the fabrication of A. mylitta SF based corneal films (Fig. 5) (Hazra et al., 2016). Epithelial cells and keratocytes from rat corneal explants (cultured on these films) not only formed cell sheets but also preserved phenotypic characters along with expression of cytokeratin3 and vimentin. The expression of ATP-binding cassette sub-family $G$ membrane 2 (ABCG2) attested the growth of limbal stem cells on the film. Implantation of the transparent and easy-to-handle biocompatible film within rabbit cornea resulted in maintenance of corneal surface 
integrity without any antagonistic effect on the intraocular pressure and electroretinogram of the implanted eyes. On the other hand, the fabrication of 2D matrices of silk gland fibroins of $A$. assamensis and A. mylitta along with cocoon fibroin of $B$. mori has also been reported recently (Kar et al., 2013). Ethanol treatment was used to induce $\beta$-sheets and impart crystallinity and insolubility. The hydrophilic films supported the attachment and spreading of MG63 human osteoblast-like cells. In parallel lines, robust membranes of $A$. mylitta middle silk gland silk sericin were found to exhibit good mechanical strength, high temperature stability, low inflammatory response (assayed using TNF- $\alpha$ release) and compatibility with

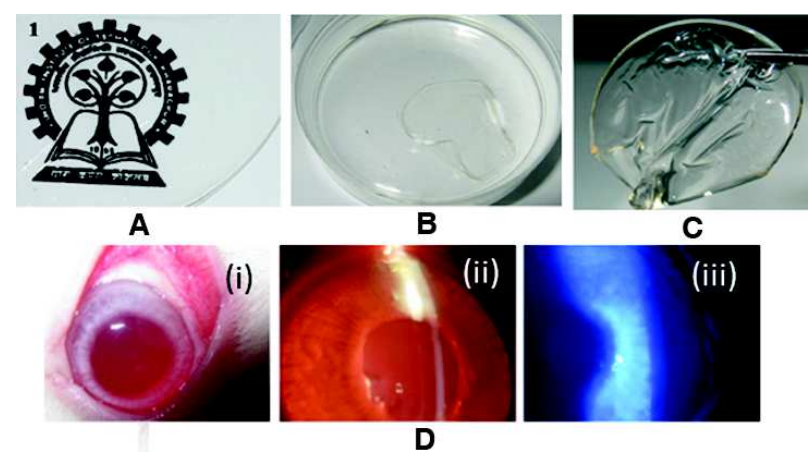

Fig. 5: Determination of transparency of $A$. mylitta films. [A] Visual appearance of $2 \mathrm{D}$ transparent thin film made of A. mylitta fibroin, the image can be clearly visualized through the transparent film. Films were [B] very thin with smooth surface and [C] easy to handle. [D] Implantation of A. mylitta films within the cornea. (i) Gross examination and (ii) slit lamp biomicroscopy showed that the film implanted within the cornea remained stable and transparent after 2 months and the films were well tolerated in vivo. (iii) Absence of fluorescein dye shows corneas were free from ulceration and erosion. Reproduced from (Hazra et al., 2016), permission sought under Creative Commons CC-BY License. ${ }^{\circledR 2016}$, Rights Managed by Nature Publishing Group

feline fibroblast cells (Dash et al., 2009).

$3 D$ matrices/scaffolds: Amongst one of the early works, biospun A. mylitta fibers (with higher stability to protease than native gland fibroin protein) were organized into macroporous 3D matrices with linear, mixed or random alignment (Fig. 6) (Mandal and Kundu, 2010). Observation of normal attachment, spreading and proliferation of feline fibroblast cells on the biospun silk matrices evinced their prospective use as cost-effective and natural substrate for regenerative medicine applications.

The potential of silk scaffolds of $A$. mylitta (nonmulberry) and B. mori (mulberry) silk gland fibroin was assessed for osteogenic differentiation of rat bone marrow cells (BMCs) (Fig. 7) (Mandal and Kundu, 2009b). High transcript levels for osteopontin, osteocalcin and osteonectin genes could be observed. High porosity and interconnectivities within pore walls of A. mylitta scaffolds allowed seeded cells to migrate uniformly, proliferate and differentiate throughout the scaffold. A. mylitta favored better osteogenesis than $B$. mori due to the former's enhanced mechanical strength.

Fibronectin like properties of $A$. mylitta SF and its prospective application as cardiac patch have also been documented (Patra et al., 2012). In this context, cardiomyocytes growing on A. mylitta scaffolds expressed connexion 43 , exhibited aligned sarcomeres and performed synchronous beating. The generated cardiac tissues were stable and spontaneously bet for at least 20 days. For prospective application in neural tissue engineering, human neural progenitor cells were found to exhibit slightly greater cell proliferation and matrix deposition on non-mulberry silk matrices compared to the mulberry counterparts. On the other hand, blended A. mylitta cocoon sericin/gelatin 3D scaffolds were found to display high compressibility, robust mechanical features, high porosity, high swellability, degradability and compatibility with feline fibroblasts (Mandal et al., 2009c). Freeze-dry technique was explored for fabricating highly interconnected porous 3D SF matrices that supported the proliferation and migration of seeded cells, eventually organizing as a functional tissue (Mandal and Kundu, 2009c). Rapid freeze-drying (at $-196^{\circ} \mathrm{C}$ ) resulted in highly interconnected pores (with $\sim 96 \%$ porosity) in sharp contrast to poor interconnectivity if slower cooling (at -20 or $-80^{\circ} \mathrm{C}$ ) was opted.

Similarly, non-bioengineered silk fibroin extracted from glands of tasar silkworms (using SDS dissolution strategy) was used to fabricate $3 \mathrm{D}$ scaffolds that supported osteogenic differentiation of human mesenchymal stem cells (hMSCs), spreading of nuclei and actin filaments with calcified nodules under single and double-seeded conditions (Fig. 8) (Mandal and Kundu, 2009d). 


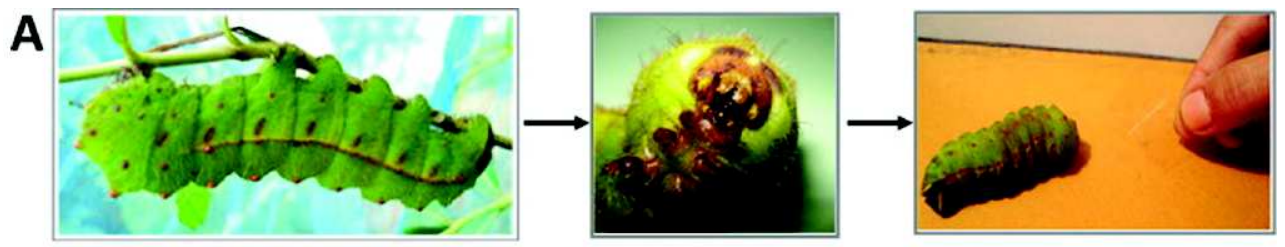

A. mylitta $5^{\text {th }}$ instar silkworm Spinneret of silkworm Biospinning on glass slides

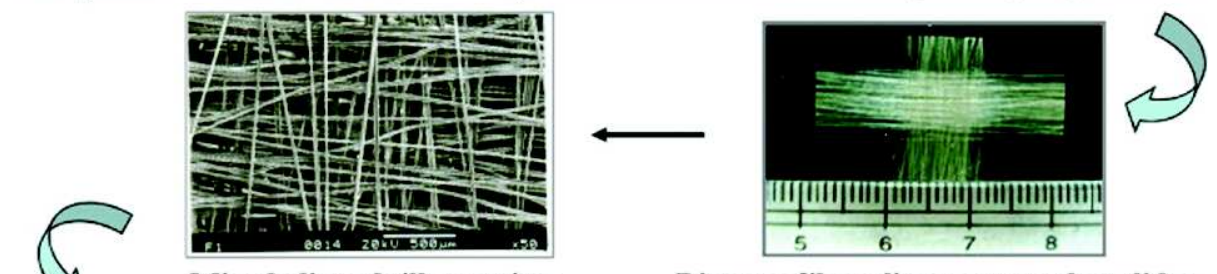

Mixed aligned silk matrices Biospun fiber alignment on glass slides

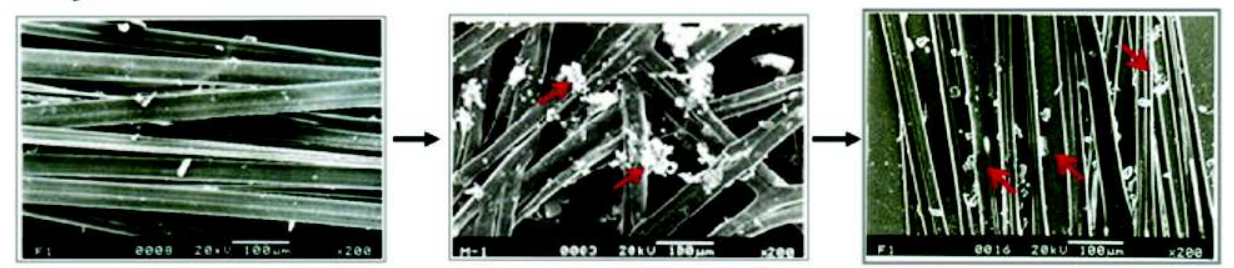

Linear aligned silk matrices Cell growth on mixed Cell growth on linear aligned fibers aligned fibers
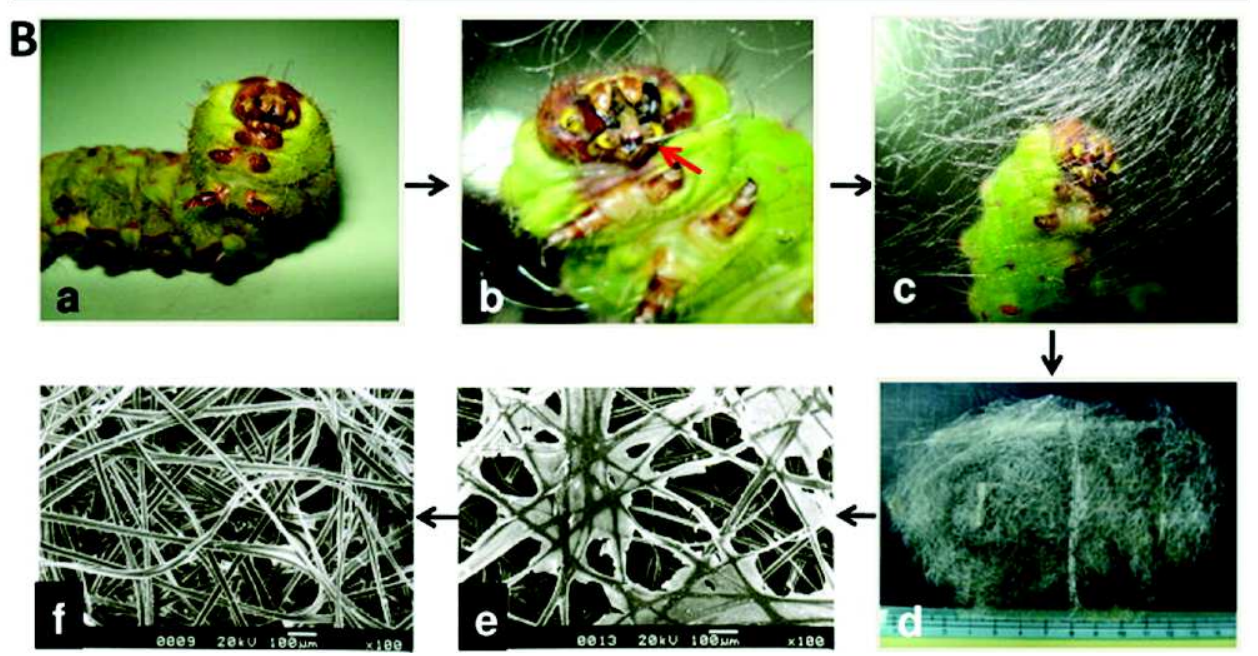

Fig. 6: Procedures followed for the fabrication of biospun matrices from silk fibroin protein obtained from fully mature fifth instar A. mylitta silkworms. [A] Images representing fabrication of linearly aligned and random fiber matrices. Scanning electron micrographs showing linearly arranged silk fiber matrix and cellular growth (day 1) on degummed random and aligned silk fiber [B] Representative images showing fabrication of randomly aligned biospun matrices. (a) silkworm at fifth instar stage; (b) biospinning of silk fibers by a silkworm using its spinneret; (c) randomly arranged silk fibers on teflon-coated glass plates, biospun by silkworm; (d) biospun mat on a flat surface; (e) profuse growth of AH 927 fibroblast cells (day 5) on biospun mat; (f) scanning electron micrographs of degummed biospun mat showing its porous nature. Reproduced with permission from (Mandal and Kundu, 2010) ${ }^{\odot 2010}$ Elsevier

In another work, SF/chitosan (CS) blended scaffolds have been projected for cartilage tissue engineering (Bhardwaj and Kundu, 2012). Expression of collagen II showed the maintenance of chondrogenesis in the constructs. SF/CS blended scaffolds and pure SF scaffolds of A. mylitta supported the highest accumulation of sulphated glycosaminoglycans (sGAG) and collagen. In a recent study, it has been substantiated that $A$. assamensis SF may serve as a befitting material for fabrication of functional scaffolds to support cartilage tissue engineering (Bhardwaj et al., 2016). Significantly 

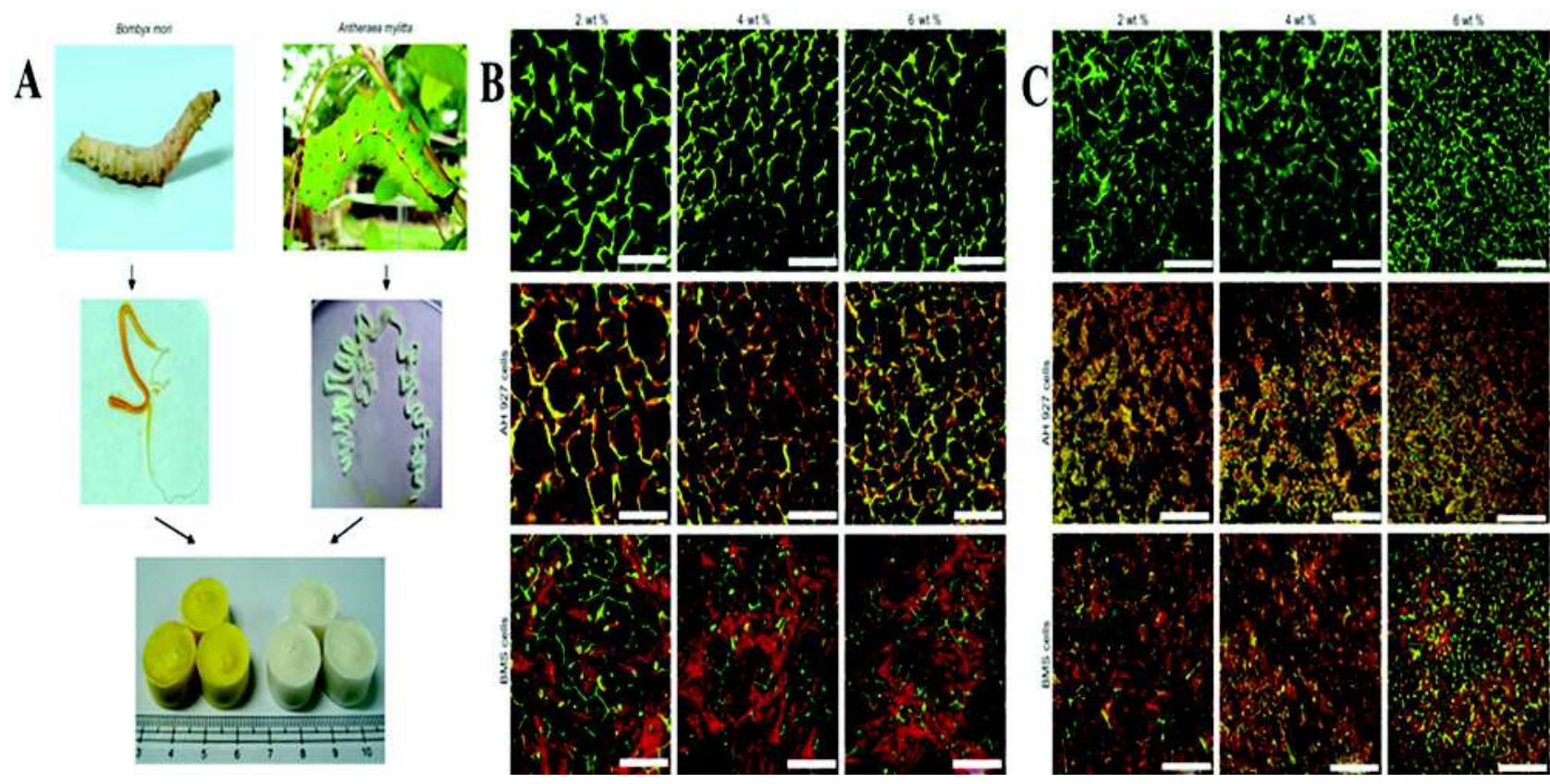

Fig. 7: [A] Schematic representation of silk fibroin source from silk glands of domesticated mulberry, B. mori and wild nonmulberry, A. mylitta silkworms for fabrication of 3D scaffolds. Confocal laser pictures showing cell growth and proliferation on 3D silk fibroin scaffolds fabricated using different wt\% of [B] A. mylitta and [C] B. mori, non-mulberry silk. Reproduced with permission from (Mandal and Kundu, 2009b) ${ }^{\odot} 2009$ Elsevier

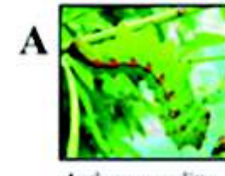

Anthenaca msditta

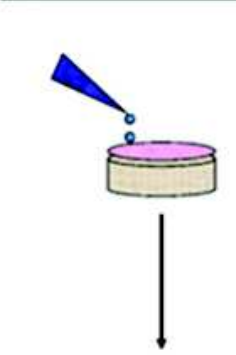

Single seeding

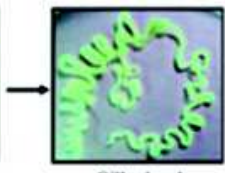

Silk gland

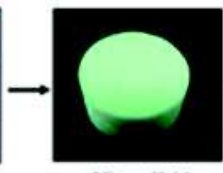

3 scaffold

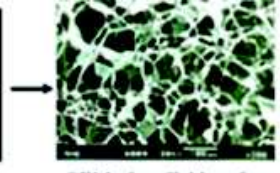

SEM of scaffold surface

Cell seeding on fibroin scaftolds
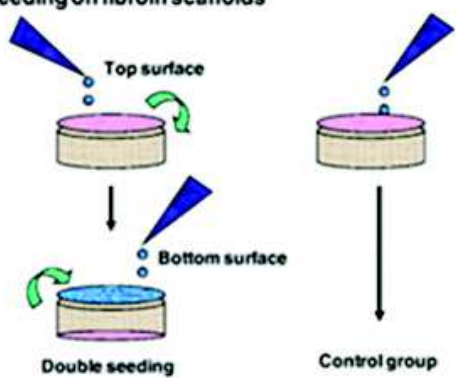

B
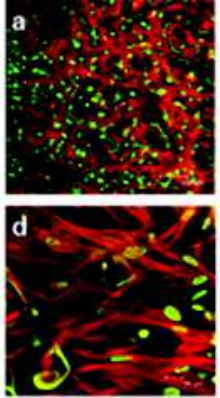
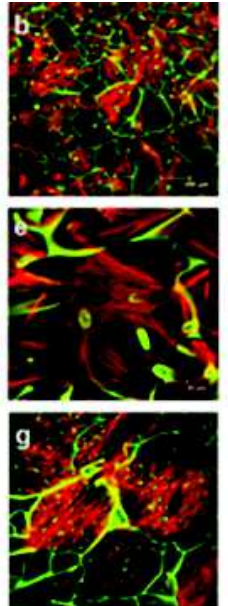
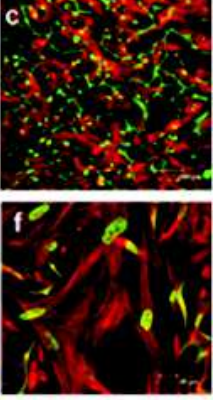

Fig. 8: [A] Schematic representation showing fabrication of 3D scaffolds using non-mulberry $A$. mylitta regenerated silk gland fibroin protein. Lower panel shows single- and double-cell-seeding techniques on silk 3D matrices. [B] Confocal laser scanning microscope images showing MSCs growth on 2 wt \% scaffolds after 28 days; seeded with $3 \times 10^{5}$ hMSCs using (a and d) double-, (b and e) single-seeding method in osteogenic media; (c and f) control media in absence of osteogenic factors; (g) double-seeded scaffold pores with entrapped cells. Cells stained with rhodamine-phalloidin for actins (red) and Hoechst 33342 for nuclei (green). Reproduced with permission from (Mandal and Kundu, 2009d) ${ }^{\circ} 2009$ Elsevier

higher accumulation of sGAG and expression of type II collagen for the $A$. assamensis $\mathrm{SF}$ scaffolds (in comparison to $P$. ricini and $B$. mori scaffolds in histochemistry and immunohistochemical studies), along with their in vivo biocompatibility post 8 weeks of implantation in a subcutaneous model of rat raise the prospects of utilizing the fabricated biomaterial for chondrocytes based cartilage repair.

\section{Electrospun Mats}

Electrospun nanofibrous mats have found prospective applications as diverse as air and water filters, drug 
loading/delivery, and tissue engineering (Konwarh et al., 2013; Bhardwaj and Kundu, 2010). In this context, non-mulberry fibroin grafted onto aminolyzed electrospun nanofibrous poly ( $\varepsilon$-caprolactone) (PCL) scaffolds have been reported to support better (in contrast to electrospun blends of SF with PCL) activity and viability of human osteoblast like cells for prospective application in bone tissue engineering (Bhattacharjee et al., 2015). It has been hypothesized that surface precipitation of nanohydroxyapatite over electrospun blends of eri-tasar nanofibrous silk fibroin scaffold (with intrinsic RGD sequence and surface hydrophilicity) might offer a prospective and costeffective approach to stimulate differentiation of stem cells without addition of growth factors (Panda et al., 2014). Mineralization studies, cell viability, expression of adhesion molecules, osteogenic differentiation and morphological visualization of differentiated cord blood human mesenchymal stem cells attested the successful endeavor of the researchers.

Researchers have fabricated a range of nonwoven scaffold composed of A. assamensis cocoon-derived silk fibroin (Kasoju et al., 2009). The scaffolds exhibited highly interconnected porous architecture with superior thermal and mechanical properties as compared to mulberry SF. The nanofibrous network was envisaged to promote enhanced cell adhesion, growth and differentiation while the microfibrous network provided adequate mechanical support. Pertinent to note is the construction of ultrathin nanofibrous film to mimic Bruch's membrane to investigate the response of human retinal pigment epithelium cells to this substrate (Xiang et al., 2014). The membrane (of thickness $\sim 3-5 \mu \mathrm{m}$ ) was fabricated from A. pernyi SF, polycaprolactone (PCL) and gelatin. Human retinal pigment epithelium (RPE) cells seeded on this membrane showed enhanced cell growth rate and expression of RPE signature genes.

Hydrogels and Sponges: Hydrogels represent a unique class of polymeric materials, the hydrophilic architecture of which endows them with large waterholding capacity in their three-dimensional networks. They have found profound utility in the biomedical realm for myriad of applications including cell encapsulation, drug delivery and so on (Hoffman, 2012). In this context, researchers delved into the prospects of using ionic liquid for the solubilization and processing of cocoon fibers of $A$. mylitta and $B$. mori to fabricate viscoelastic, degradable hydrogels (Silva et al., 2013). The scientists had reported the adherence and migration of metabolically active human adipose derived stem cells (hASCs) at various levels within the hydrogels. Transforming growth factor $\beta 1$ (TGF- $\beta 1$ ) loaded cross-linked hydrogel matrices, fabricated using silk cocoon sericin of A. mylitta and carboxymethyl cellulose, were shown to provide a 3D cell-interactive environment to human keratinocytes (Nayak and Kundu, 2014). Its potential use has been proposed for cell wound dressing material. In a recent study, A. assama silk fibroinagarose blended porous hydrogels with adequate elasticity have been forwarded as a better choice in comparison to the mulberry counterpart for cartilaginous tissue formation (Singh et al., 2016). The study evinced a simple design strategy using silk fibroin to abrogate the undesirable features of agarose (otherwise considered the gold standard biomaterial in cartilage tissue engineering) including immunogenicity, non-degradability, low amenability to customization and low cell adhesiveness. The immunocompatible blended hydrogels supported high levels of sulphated glycosaminoglycans and collagen apart from up-regulation of cartilage specific aggrecan and sox-9 along with collagen type II marker genes.

Furthermore, researchers have fabricated cocoon derived eri silk sponges, exhibiting flexibility to adapt and recover shapes depending on applied load as well as sustained release of a model drugibuprofen. The sponges supported the in vitro cellular adhesion, proliferation and ECM production by ATDC5 chondrocyte-like cells (Silva et al., 2016).

Micromolded Matrices : Spatiotemporally tuned patterns of micrometer dimensions fetch numerous advantages over macro scale cultures, particularly when it comes to presenting cells with accurate and controlled cues of biologically relevant macromolecules (including extracellular signaling components, growth factors and hormones) (Folch and Tourovskaia, 2007). Though use of materials of biological origin for preparing micromolded tissue engineering scaffolds is preferred over synthetic materials owing to easy bio-recognition and low toxicity, the practical utility is hindered by their innate poor physical properties, mechanical attributes and low processibility (Langer and Tirrell, 2004). In this 
context, a micromolded matrix was fabricated using A. mylitta regenerated silk fibroin casted over the negative molds of polydimethylsiloxane (PDMS) (Mandal et al., 2009b). Uniform spreading and proliferation of AH927 (fibroblasts of murine origin) with spindle shaped morphology and actin filaments running across the micromolded surface covering the nucleus were observed. It is expected that fabrication of such silk micromolded matrices (with RGD sequences that allow better adherence and proliferation of cells) for studying cell-surface interactions would be easier than polystyrene material.

\section{Model-molecule/drug Delivery}

Nanomaterials with unique shape-size-surface chemistry accord have been used in diverse domains like catalysis, biomolecular trapping, drug delivery and so on (Konwarh et al., 2009; Konwarh et al., 2012). In this context, the self-assembled micellar nanostructures (100-110 nm) of A. mylitta silk sericin blended with pluronic F-127 and F-87 were reported for delivery of hydrophilic (FITC-inulin) and hydrophobic (paclitaxel, anticancer drug) drugs to target sites (Mandal and Kundu, 2009e). Up-regulation of Bax (pro-apoptotic protein), down-regulation of Bcl-2 (anti-apoptotic protein) and cleavage of poly ADP ribose polymerase (PARP, regulatory protein) attested the nanoparticle loaded drug-induced apoptosis in MCF-7 cells. On the other hand, a simple design approach based on incorporating proteinimmobilized 3D calcium alginate beads within silk fibroin was effectively employed to separately tailor the release rate of two different model proteins- BSA and FITC-Inulin (Mandal and Kundu, 2009a). It was observed that the sustained release profile of the encapsulated model drugs was dictated by their respective molecular weight. Such multiple drug releasing matrices could not only be used for tuning release profiles, but also employed to guide the differentiation of stem cells (MSCs) into different lineages.

A. mylitta silk fibroin nanoparticles conjugated to folate (a vitamin) have been reported as nontoxic nanocarriers to facilitate targeted and sustained release of doxorubicin in cancer cells (Subia et al., 2014). Furthermore, the fabrication (using a combination of wet-milling and spray drying techniques) of non-mulberry (A. assamensis, A. mylitta and $P$. ricini) silk fibroin microparticles has been reported for prospective drug delivery application (Bhardwaj et al., 2015). Relevant to note is the fact that these microparticles assisted greater cell adhesion, spreading and viability of mouse fibroblasts in comparison to $B$. mori fibroin microparticles. Issues of inflammation, loosening and bacterial infection are common confrontation in metal based implants used for orthopaedic/dental surgeries. In this context, gentamicin loaded A. mylitta SF nanoparticles deposited over titanium (Ti) surface not only supported osteoblast adhesion, proliferation and differentiation with respect to bare Ti surface but also exhibited sustained in vitro drug release (Sharma et al., 2016). Fibroin from both mulberry and non-mulberry silkworm sources has been used for surface modification of titanium alloy (Naskar et al., 2014). The Ti surfaces with immobilized non-mulberry fibroin showed better results for cell adherence, differentiation and mineralization.

\section{Suture and Antimicrobial Materials}

A recent research endeavour has attested excellent mechanical properties, antimicrobial behaviour and wound healing activity of plasma polypropylene grafted muga silk as sterilized suture (a medical device applied to hold body tissues together post an injury or surgery) (Gogoi et al., 2014). In another of their works, plasma induced grafting of chitosan followed by fortification with penicillin forwarded $A$. assamensis SF yarn as an antibacterial (against Gram positive and Gram negative bacteria) and antithrombogenic material (Choudhury et al., 2016).

\section{In vitro Tumour Model}

Non-mulberry silk fibroin based 3D systems have also been proposed as in vitro tumor model for assessment of anticancer drug activity. In this context, cytotoxic effects of Paclitaxel, Celecoxib and ZD6474 at various concentrations have been assessed for breast cancer line MDA-MB-231 cells cultured on A. mylitta fibroin based 2D films and 3D scaffold (Talukdar and Kundu, 2012). Interestingly, the researchers reported the need of higher drug concentrations to attain a comparable decrement in cell viability and invasive potential in 3D culture. Additionally, the effect of innate attributes of tasar silk fibroin has been assessed on the HepR21 cells (that overexpress hyaladherin, hyaluronan-binding protein 1) for developing an in vitro 3D multicellular model to understand the progression 
of hepatocarcinoma (Kundu et al., 2013). The researchers reported comparable reduction of the tumour potency associated with the augmented pool of hyaluronic acid (HA, a key player in tumour progression and metastasis) and HA cables on application of 4-Methylumbelliferone (4-MU) (an inhibitor of hyaluronan synthase) in 2D and 3D models.

The works that have been highlighted here are a non-exhaustive list. Reports on novel endeavours on the use of non-mulberry silk for various biomedical applications are constantly escalating.

\section{Conclusion}

Tapping the under-explored potential of non-mulberry silk may open up new horizons in the endeavours to fabricate novel biomaterials for translational medicine. Resorting to various novel and benign extraction protocols of the silk proteins, scientists have fabricated various formats of non-mulberry silk biomaterial (scaffolds, films, hydrogels, nanoparticles, microspheres, nanofibrous mats) for applications in regenerative medicine and $3 \mathrm{D}$ disease tissue modelling. Silk based cost effective tissue engineered constructs are being designed with the aim of restoring biochemical and biomechanical functions of the native tissue. Such biomaterials may also fetch immense application in cell-encapsulation and delivery of various biomolecular cargoes (e.g., hydrophilic and

\section{References}

Abbott R D and Kaplan D L (2016) Engineering biomaterials for enhanced tissue regeneration Curr Stem Cell Rep 2 140146

Bellis S L (2011) Advantages of RGD peptides for directing cell association with biomaterialsBiomaterials 32 4205-4210

Bhardwaj N and Kundu S C (2012) Chondrogenic differentiation of rat MSCs on porous scaffolds of silk fibroin/chitosan blends Biomaterials 33 2848-2857

Bhardwaj N and Kundu S C (2010) Electrospinning: A fascinating fiber fabrication technique Biotechnol Adv 28 325-347

Bhardwaj N, Rajkhowa R, Wang X and Devi D (2015) Milled non-mulberry silk fibroin microparticles as biomaterial for biomedical applications Int J Biol Macromol 81 31-40

Bhardwaj N, Singh Y P, Devi D, Kandimalla R, Kotoky J and Mandal B B (2016) Potential of silk fibroin/chondrocyte constructs of muga silkworm Antheraea assamensis for hydrophobic drugs). However, a deeper insight into the action at the bio-interface is prerequisite for the clinical translation and availability of the non-mulberry silk based 'off-the-shelf' biomedical products. Besides, exploitation of non-mulberry silk sericin in domains like development of personal care products could open up novel profitable avenues. This would further catalyse the building up of scientific repository and creation of non-mulberry-silk based scientific innovations for practical biomedical applications.

\section{Acknowledgment}

BBM thankfully acknowledges the receipt of funding support from Department of Biotechnology (DBT), Department of Science and Technology (DST), Indian Council of Medical Research (ICMR) and Department of Atomic Energy (DAE), Government of India. The receipt of institutional post-doctoral fellowship from Indian Institute of Technology Guwahati is thankfully acknowledged by RK. The authors express gratitude to Mr. Prerak Gupta, Mr. Omkar Majumder, Mr. Joseph Christakiran and Miss Janani Guru for their useful suggestions.

\section{Conflict of Interest}

The authors declare that there exists no conflict of interest.

cartilage tissue engineering J Mater Chem B 4 3670-3684

Bhardwaj N, Sow W T, Devi D, Ng K W, Mandal B B and Cho N J (2015) Silk fibroin-keratin based 3D scaffolds as a dermal substitute for skin tissue engineering Integr Biol 7 53-63

Bhattacharjee P, Naskar D, Kim H W, Maiti T K, Bhattacharya D and Kundu S C (2015) Non-mulberry silk fibroin grafted PCL nanofibrous scaffold: Promising ECM for bone tissue engineering Eur Polym J 71 490-509

Chae S K, Kang E, Khademhosseini A and Lee S H (2013) Micro/ nanometer scale fiber with highly ordered structures by mimicking the spinning process of silkworm Adv Mater 25 3071-3078

Choudhury A J, Gogoi D, Kandimalla R, Kalita S, Chaudhari Y B, Khan M R, Kotoky J and Chutia J (2016) Penicillin impregnation on oxygen plasma surface functionalized chitosan/Antheraea assama silk fibroin: Studies of antibacterial activity and antithrombogenic property Mater Sci Eng C 60 475-484 
Dash B C, Mandal B B and Kundu S C (2009) Silk gland sericin protein membranes: Fabrication and characterization for potential biotechnological applications J Biotechnol 144 321-329

Datta A, Ghosh A K and Kundu S C (2001) Purification and characterization of fibroin from the tropical saturniid silkworm, Antheraea mylittaInsect Biochem Mol Biol 31 1013-1018

Folch A and Tourovskaia A (2007) Therapeutic Micro/Nano Technology, Springer

Gil E S, Mandal B B, Park S H, Marchant J K, Omenetto F G and Kaplan D L (2010) Helicoidal multi-lamellar features of RGD-functionalized silk biomaterials for corneal tissue engineering Biomaterials 31 8953-8963

Gogoi D, Choudhury A J, Chutia J, Pal A R, Khan M, Choudhury M, Pathak P, Das G and Patil D S (2014) Development of advanced antimicrobial and sterilized plasma polypropylene grafted muga (Antheraea assama) silk as suture biomaterial Biopolymers 101 355-365

Gupta A, Mita K, Arunkumar K P and Nagaraju J (2015) Molecular architecture of silk fibroin of Indian golden silkmoth, Antheraea assamaSci Rep 512706

Gupta P, Kumar M, Bhardwaj N, Kumar J P, Krishnamurthy C S, Nandi S K and Mandal B B (2016) Mimicking form and function of native small diameter vascular conduits using mulberry and non-mulberry patterned silk films ACS Appl Mater Inter 8 15874-15888

Gurtner G C, Callaghan M J and Longaker M T (2007) Progress and potential for regenerative medicine Annu Rev Med $\mathbf{5 8}$ 299-312

Hazra S, Nandi S, Naskar D, Guha R, Chowdhury S, Pradhan N, Kundu S C and Konar A (2016) Non-mulberry silk fibroin biomaterial for corneal regeneration Sci Rep 621840

Hoffman A S (2012) Hydrogels for biomedical applications $A d v$ Drug Deliv Rev 64 18-23

Inoue S, Tanaka K, Arisaka F, Kimura S, Ohtomo K and Mizuno S (2000) Silk fibroin of Bombyx mori is secreted, assembling a high molecular mass elementary unit consisting of $\mathrm{H}$ chain, L-chain, and p25, with a 6: 6: 1 molar ratio J Biol Chem 275 40517-40528

Kar S, Talukdar S, Pal S, Nayak S, Paranjape P and Kundu S (2013) Silk gland fibroin from indian muga silkworm Antheraea assama as potential biomaterial Tissue Eng Regen Med 10 200-210

Kasoju N, Bhonde R R and Bora U (2009) Fabrication of a novel micro-nano fibrous nonwoven scaffold with Antheraea assama silk fibroin for use in tissue engineering Mater Lett $632466-2469$
Konwarh R, Gupta P and Mandal B B (2016) Silk-microfluidics for advanced biotechnological applications: A progressive review. Biotechnol Adv 34 845-858

Konwarh R, Karak N, Misra M (2013) Electrospun cellulose acetate nanofibers: The present status and gamut of biotechnological applications Biotechnol Adv 31 421-437

Konwarh R, Karak N, Rai S K and Mukherjee A K (2009) Polymer-assisted iron oxide magnetic nanoparticle immobilized keratinase Nanotechnol 20225107

Konwarh R, Pramanik S, Devi K S P, Saikia N, Boruah R, Maiti T K, Deka R C and Karak N (2012) Lycopene coupled 'trifoliate' polyaniline nanofibers as multi-functional biomaterial J Mater Chem 22 15062-15070

Kundu B, Saha P, Datta K and Kundu S C (2013) A silk fibroin based hepatocarcinoma model and the assessment of the drug response in hyaluronan-binding protein 1 overexpressed HepG2 cells Biomaterials 34 9462-9474

Kundu S, Kundu B, Talukdar S, Bano S, Nayak S, Kundu J, Mandal B B, Bhardwaj N, Botlagunta M and Dash B C (2012) Non-mulberry silk biopolymers Biopolymers 97 455-467

Langer R and Tirrell D A (2004) Designing materials for biology and medicine Nature $\mathbf{4 2 8}$ 487-492

Li G, Li Y, Chen G, He J, Han Y, Wang X and Kaplan D L (2015) Silk-based biomaterials in biomedical textiles and fiberbased implants Adv Healthc Mater 4 1134-1151

Lieben L (2016) Regenerative medicine: The future of 3D printing of human tissues is taking shape Nat Rev Rheumatol 12 191

Liu W, Wang Y, Yao J, Shao Z and Chen X (2016) Tamoxifenloaded silk fibroin electrospun fibers Mater Lett 178 31-34

Malay A D, Kenjiro Y, Watanabe H, Sato R, Ifuku N, Masunaga H, Hikima T, Guan J, Mandal B B, Damrongsakkul S and Numata K (2016) Relationships between physical properties and sequence in silkworm silks Sci Rep 627573

Mandal B B and Kundu S C (2008) A novel method for dissolution and stabilization of non mulberry silk gland protein fibroin using anionic surfactant sodium dodecyl sulfate Biotechnol Bioeng 99 1482-1489

Mandal B B and Kundu S C (2010) Biospinning by silkworms: Silk fiber matrices for tissue engineering applications Acta Biomater 6 360-371

Mandal B B and Kundu S C (2009a) Calcium alginate beads embedded in silk fibroin as 3D dual drug releasing scaffolds Biomaterials 30 5170-5177

Mandal B B and Kundu S C (2009b) Osteogenic and adipogenic differentiation of rat bone marrow cells on non-mulberry 
and mulberry silk gland fibroin 3D scaffolds Biomaterials 30 5019-5030

Mandal B B and Kundu S C (2009c) Cell proliferation and migration in silk fibroin 3D scaffolds Biomaterials 30 2956-2965

Mandal B B and Kundu S C (2009d) Non-mulberry silk gland fibroin protein 3D scaffold for enhanced differentiation of human mesenchymal stem cells into osteocytes Acta Biomater 5 2579-2590

Mandal B B and Kundu S C (2009e) Self-assembled silk sericin/ poloxamer nanoparticles as nanocarriers of hydrophobic and hydrophilic drugs for targeted delivery Nanotechnol 20355101

Mandal B B, Das S, Choudhury K and Kundu S C (2010) Implication of silk film RGD availability and surface roughness on cytoskeletal organization and proliferation of primary rat bone marrow cells Tissue Eng Part A 16 2391-2403

Mandal B B, Mann J K and Kundu S C (2009a) Silk fibroin/ gelatin multilayered films as a model system for controlled drug release Eur J Pharm Sci 37 160-171

Mandal B B, Das T and Kundu S C (2009b) Non-bioengineered silk gland fibroin micromolded matrices to study cell-surface interactions Biomed Microdevices 11 467-476

Mandal B B, Priya A S and Kundu S C (2009c) Novel silk sericin/ gelatin 3D scaffolds and 2D films: Fabrication and characterization for potential tissue engineering applications Acta Biomater 5 3007-3020

Mandal B B, Gil E S, Panilaitis B and Kaplan D L (2013) Laminar silk scaffolds for aligned tissue fabrication Macromol Biosci 13 48-58

Mandal B B, Grinberg A, Seok Gil E, Panilaitis B and Kaplan D L (2012) High-strength silk protein scaffolds for bone repair. Proc Nat Acad Sci USA 109 7699-7704

Mandal B B, Park S H, Gil E S and Kaplan D L (2011) Multilayered silk scaffolds for meniscus tissue engineering Biomaterials 32 639-651

Mason C and Dunnill P (2008) A brief definition of regenerative medicine Regen Med 3 1-5

Melke J, Midha S, Ghosh S, Ito K and Hofmann S (2016) Silk fibroin as biomaterial for bone tissue engineering Acta Biomater 31 1-16

Naskar D, Nayak S, Dey T and Kundu S C (2014) Non-mulberry silk fibroin influence osteogenesis and osteoblastmacrophage cross talk on titanium based surface Sci Rep 4 4745

Nayak S and Kundu S (2014) Sericin-carboxymethyl cellulose porous matrices as cellular wound dressing material $J$
Biomed Mater Res Part A 102 1928-1940

Panda N, Bissoyi A, Pramanik K and Biswas A (2014) Directing osteogenesis of stem cells with hydroxyapatite precipitated electrospun eri-tasar silk fibroin nanofibrous scaffold $J$ Biomater Sci, Polym Ed 25 1440-1457

Patil R, Naika H R, Rayar S, Balashanmugam N, Uppar V and Bhattacharyya A (2016) Green synthesis of gold nanoparticles: Its effect on cocoon and silk traits of mulberry silkworm (Bombyx mori L.) Part Sci Technol DOI: $10.1080 / 02726351.2016 .1154121$

Patra C, Talukdar S, Novoyatleva T, Velagala S R, Mühlfeld C, Kundu B, Kundu S C and Engel FB (2012) Silk protein fibroin from Antheraea mylitta for cardiac tissue engineering Biomaterials 33 2673-2680

Phiel C J and Klein P S (2001) Molecular targets of lithium action Annu Rev Pharmacol Toxicol 41 789-813

Phukan R D (2012) Muga silk industry of Assam in historical perspectives GJHSS 12 5-8

Reddy N and Yang Y (2010) Structure and properties of ultrafine silk fibers produced by Theriodopteryx ephemeraeformis J Mater Sci 45 6617-6622

Sangkert S, Kamonmattayakul S, Chai W L and Meesane J (2016) A biofunctional-modified silk fibroin scaffold with mimic reconstructed extracellular matrix of decellularized pulp/ collagen/fibronectin for bone tissue engineering in alveolar bone resorption Mater Lett 166 30-34

Sen K and Babu K (2004) Studies on Indian silk. I. Macrocharacterization and analysis of amino acid composition J Appl Pol Sci 92 1080-1097

Sharma S, Bano S, Ghosh A S, Mandal M, Kim H W, Dey T and Kundu S C (2016) Silk fibroin nanoparticles support in vitro sustained antibiotic release and osteogenesis on titanium surface Nanomed Nanotechnol Biol Med 12 11931204

Silva S S, Oliveira N M, Oliveira M B, Da Costa D P S, Naskar D, Mano J F, Kundu S C and Reis R L (2016) Fabrication and characterization of eri silk fibers-based sponges for biomedical application Acta Biomater 32 178-189

Silva S S, Popa E G, Gomes M E, Oliveira M B, Nayak S, Subia B, Mano J F, Kundu S C and Reis R L (2013) Silk hydrogels from non-mulberry and mulberry silkworm cocoons processed with ionic liquids Acta Biomater 9 8972-8982

Singh Y P, Bhardwaj N and Mandal B B (2016) Potential of agarose/silk fibroin blended hydrogel for in vitro cartilage tissue engineering ACS Appl Mater Interfaces 8 2123621249

Subia B, Chandra S, Talukdar S and Kundu S C (2014) Folate 
conjugated silk fibroin nanocarriers for targeted drug delivery Integr Biol 6 203-214

Talukdar S and Kundu S C (2012) A non mulberry silk fibroin protein based 3D in vitro tumor model for evaluation of anticancer drug activity Adv Funct Mater 22 4778-4788

Tien L W, Gil E S, Park S H, Mandal B B and Kaplan D L (2012) Patterned silk film scaffolds for aligned lamellar bone tissue engineering Macromol Biosci 12 1671-1679

Wang Y, Kong Y, Zhao Y, Feng Q, Wu Y, Tang X, Gu X and Yang Y (2016) Electrospun, reinforcing network-containing, silk fibroin-based nerve guidance conduits for peripheral nerve repair J Biomater Tissue Eng 6 53-60

Xiang P, Wu K C, Zhu Y, Xiang L, Li C, Chen D L, Chen F, Xu G, Wang A and Li M (2014) A novel Bruch's membranemimetic electrospun substrate scaffold for human retinal pigment epithelium cells Biomaterials 35 9777-9788

Zhou C Z, Confalonieri F, Jacquet M, Perasso R, Li Z G and Janin J (2001) Silk fibroin: Structural implications of a remarkable amino acid sequence Proteins: Struct Funct Bioinf 44 119-122. 